\title{
Structure and Magnetization of Small Monodisperse Platinum Clusters
}

\author{
Xiong Liu, Matthias Bauer, Helmut Bertagnolli, and Emil Roduner* \\ Institut für Physikalische Chemie, Universität Stuttgart, Pfaffenwaldring 55, D-70569 Stuttgart, Germany \\ Joris van Slageren \\ 1. Physikalisches Institut, Universität Stuttgart, Pfaffenwaldring 57, D-70550 Stuttgart, Germany \\ Fritz Phillipp \\ Max Planck Institut für Metallforschung, Heisenbergstrasse 3, D-70569 Stuttgart, Germany
}

(Received 1 May 2006; published 18 December 2006)

\begin{abstract}
Magnetization measurements of well-characterized monodisperse Pt clusters consisting of $13 \pm 2$ atoms in a zeolite confirm the predicted extraordinary magnetic polarization with up to 8 unpaired electrons on a cluster, corresponding to a magnetic moment of $0.65(5) \mu_{B}$ per atom. The effect is partly quenched by hydrogen chemisorption. The study provides insight into the electronic structure of the cluster and is fundamental for an understanding of how magnetism develops in small clusters.
\end{abstract}

DOI: 10.1103/PhysRevLett.97.253401

PACS numbers: $36.40 . \mathrm{Cg}, 36.40 . \mathrm{Mr}, 61.10 . \mathrm{Ht}, 61.46 . \mathrm{Bc}$

While enhanced magnetism in clusters of elements that are ferromagnetic as bulk solids is well known and has been demonstrated in Stern-Gerlach deflection experiments [1], theoretical studies predicted high-spin ground states for clusters of up to 13 atoms also of Pd and Pt, i.e., of elements which do not show magnetic ordering in the bulk [2-4]. Nanoparticles comprising several hundred atoms of $\mathrm{Au}, \mathrm{Pd}$, and $\mathrm{Pt}$ embedded in a polymer revealed magnetic moments corresponding to several unpaired electron spins per entire particle $[5,6]$, but no experimental studies exist for smaller clusters. The electronic structure of small metal clusters is a strong function of size [7]. This is because the average energy spacing of electronic states at the Fermi level, the Kubo gap $\delta N$, scales as $\Delta / N$, where $N$ is the number of atoms in the cluster. $\Delta$ is the width of the valence band which is only a weak function of size and assumes typically values on the order of a few eV. For a given value of $N$ the cluster undergoes a thermal transition from an insulator to a semiconductor at the temperature where the valence electrons can overcome the Kubo gap, providing the band is incompletely filled with electrons. Alternatively, for a given temperature this transition can occur as a function of $N$. Furthermore, when $\delta N$ is sufficiently small, electrons will occupy the levels with parallel spins, following Hund's rule [3], which leads to a high spin state that is accompanied with a high magnetic moment. But when $\delta N$ exceeds the spin pairing energy the system switches to a low spin state. On this basis, a small cluster could be expected to be in a low spin state, because of its relatively large value of $\delta N$. However, the picture is complicated further by symmetry. A high symmetry may lead to a highly degenerate HOMO (highest occupied molecular orbital), favoring high spin states. When the symmetry is broken, degeneracy is lifted and the ground state may be one of lower spin, depending on the extent to which the symmetry is broken. Additional complications can arise in applied magnetic fields when the Zeeman or spin-orbit energies compete with level splittings at the Fermi level, leading to magnetic field and also temperature dependent spin states. Furthermore, when the surface is capped, each chemisorbed species engages one of the potential conduction electrons of the cluster and pins them in a localized chemical bond.

It is vis-à-vis this complex background that the present results with Pt clusters have to be seen. Atomic Pt has the electron configuration $[\mathrm{Xe}] 4 f^{14} 5 d^{9} 6 s^{1}$ and a spin triplet ground state. In the bulk metal the $6 s$ atomic orbitals are diffuse, and the $s$ band is broad. It is the $5 d$ band which is mainly engaged in bonds to chemisorbed molecules and when electrons are removed or added. Density functional calculations for $\mathrm{Pt}_{13}$ found several distorted or even amorphous cluster geometries with energies lower by as much as $0.16 \mathrm{eV}\left(1300 \mathrm{~cm}^{-1}\right)$ than those of an ideal icosahedron or cuboctahedron $[3,8]$, also in the presence of chemisorbed hydrogen [4].

The synthesis of small Pt clusters of 13-20 atoms in zeolite Y was reported previously [9], but there were no magnetic measurements. Here, Pt clusters were prepared in $\mathrm{NaY}$ zeolite by ion exchange with $3 \mathrm{mM}$ aqueous $\left[\mathrm{Pt}\left(\mathrm{NH}_{3}\right)_{4}\right] \mathrm{Cl}_{2}$ solution, followed by stirring at $343 \mathrm{~K}$ for at least $48 \mathrm{~h}$. Chemical analysis confirmed that this procedure leads to a Pt loading of ca. $6 \mathrm{wt} \%$. The sample was washed, dried, and calcined in flowing $\mathrm{O}_{2}$ at a heating rate of $0.5 \mathrm{~K} \cdot \mathrm{min}^{-1}$ between 295 and $573 \mathrm{~K}$ and holding $573 \mathrm{~K}$ for $5 \mathrm{~h}$. Reduction was then performed in flowing hydrogen $\left(100 \mathrm{ml} \cdot \min ^{-1} \mathrm{~g}^{-1}\right)$ with a heating rate of $6 \mathrm{~K} \cdot \mathrm{min}^{-1}$ between 293 and $473 \mathrm{~K}$ and keeping it at $473 \mathrm{~K}$ for $1 \mathrm{~h}$. For hydrogen desorption, a glass tube containing $\mathrm{Pt} / \mathrm{NaY}$ powder was connected to a turbomolecular pump, evacuated, and heated in a small oven at $393 \mathrm{~K}$ for about $120 \mathrm{~min}$. All sample transfer was carried out in a glove box under $\mathrm{N}_{2}$. 
Characterization using a JEOL 4000FX transmission electron microscope with a point resolution of $0.2 \mathrm{~nm}$ at an acceleration voltage of $400 \mathrm{kV}$ confirmed that Pt was present in very high dispersion with a cluster size on the order of $1 \mathrm{~nm}$, but the clusters were labile and rearranged under the influence of the energetic electron beam, preventing a more precise size or structure determination. In such cases extended $\mathrm{x}$-ray absorption fine structure (EXAFS) spectroscopy provides detailed information by analysis of the first nearest neighbor shell [10]. Measurements were conducted at the Pt $L_{3}$ edge at the beam line $A 1$ at HASYLAB/Desy (Hamburg) at liquid nitrogen temperature to reduce information loss due to thermal distortion by cutoff of the low $k$ range [11]. By comparison with the bulk Pt reference the x-ray absorption near edge structure (XANES) region proves an oxidation state zero within experimental error for the samples investigated. Compared with the bulk spectrum the background corrected absorption spectra show a drastic intensity reduction (Fig. 1). This effect of very small cluster size is even more obvious in the Fourier transforms of the $k^{2}$-weighted $\chi(k)$ spectra. Only one Pt shell can be resolved, and its intensity is reduced by a factor of 10 compared to the bulk (Fig. 2). The Pt coordination numbers found in a fit of a single-Pt-shell are 5.4 and 5.6 for the $\mathrm{H}_{2}$ adsorbed and desorbed samples, respectively. This coordi-
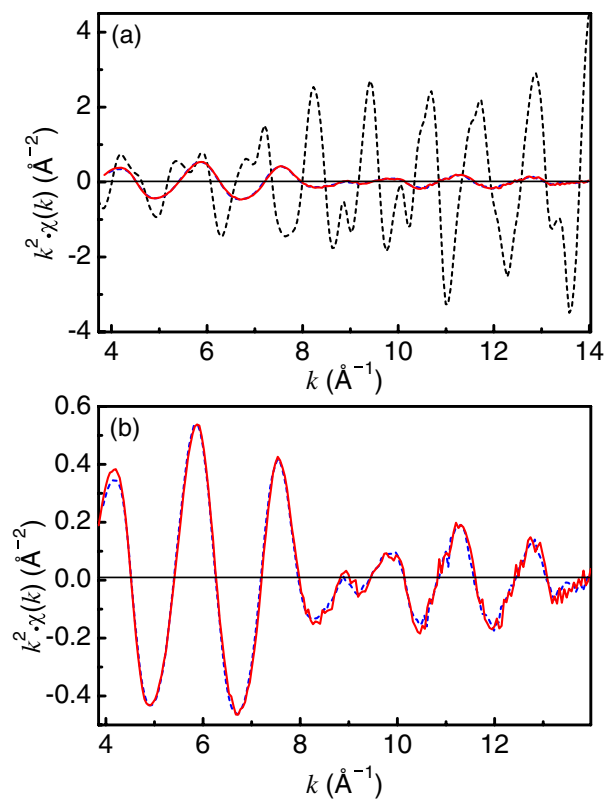

FIG. 1 (color online). (a) Comparison of the $k^{2}$-weighted $\chi(k)$ functions of the $\mathrm{H}_{2}$-desorbed (solid line) samples in comparison with bulk Pt (dashed line). Background correction was carried out using the AUTOBK program package [10]. As a result of the very small particle size, the oscillatory fine structure of the nanoparticles is completely different from that of bulk $\mathrm{Pt}$; (b) spectra of $\mathrm{H}_{2}$-adsorbed (dotted line) and $\mathrm{H}_{2}$-desorbed (solid line) samples in detailed comparison. From the identity of the two spectra it can be concluded that no structural changes are induced by hydrogen adsorption. nation number corresponds to a cluster size of $13 \pm 2 \mathrm{Pt}$ atoms and a diameter of $7 \pm 1 \AA[12,13]$. The presence of a significant fraction of larger particles can be excluded as they would dominate the coordination number of the first shell, leading to higher values [10]. Two distinct Pt shells could be resolved in a more detailed fit of the experimental data, which resulted in an improved fit index by more than $20 \%$. The obtained coordination numbers are in good agreement with the expected values of 1.8 and 4.6 for an ideal icosahedron. The mismatch between the modulo function and imaginary part in the Fourier transform proves strong anharmonic contributions to the EXAFS function, which were accounted for by inclusion of a higher cumulant to avoid errors in the distance determination [11]. The obtained distances are in good agreement with model calculations, which show an obvious contraction in comparison to bulk Pt. The Debye-Waller factor of the surface atoms is more than twice the value of the bulklike central atom in the icosahedra. The high value for the mean-square displacement can partly also be due to high disorder caused by small structural deviations from the ideal icosahedral symmetry, which are obtained as multiple energy minima in atomistic calculations [4]. A three-atom oxygen shell is attributed to the zeolite matrix [14], as an oxidation of the clusters can be excluded from XANES considerations. The finding is compatible with a situation in which the cluster sits on a puckered 6-ring window (SII site of the faujasite structure) in which three
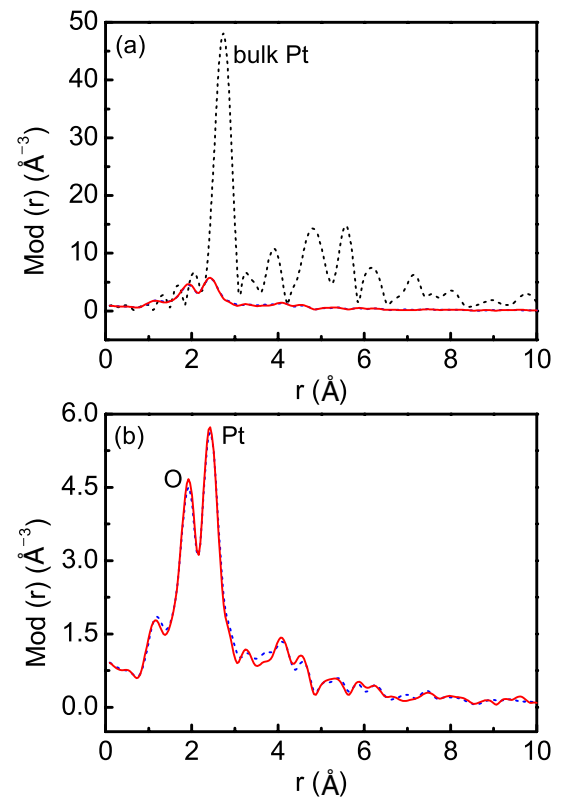

FIG. 2 (color online). (a) Fourier transforms of the $\mathrm{H}_{2}$-adsorbed (dotted line) and $\mathrm{H}_{2}$-desorbed (solid line) samples in comparison with bulk Pt (dashed line). The intensity loss and distance contraction of the Pt shell at $2.6 \AA$ due to extremely small cluster size is obvious. At $2.0 \AA$ the oxygen shell is observed; (b) shows the enlarged spectra of the samples containing nanoclusters. 
oxygen atoms point towards the cluster and three away from it.

Magnetization measurements were performed using a superconducting quantum interference device (SQUID, quantum design MPMXL7). All data are reported per mol Pt atoms and corrected by subtraction of the magnetization of the same amount of original zeolite in the same capsule. Above $200 \mathrm{~K}$ the data suggest a temperatureindependent susceptibility $\chi_{0}$ of ca. $+1.0 \times 10^{-4} \mathrm{emu}$. $\mathrm{mol}^{-1}$ and $+4.0 \times 10^{-4} \mathrm{emu} \cdot \mathrm{mol}^{-1}$ for the original and the hydrogen-desorbed samples, respectively (see discussion below). For comparison, the Pauli paramagnetic susceptibility of bulk platinum corresponds to $+1.9 \times 10^{-4} \mathrm{emu} \cdot \mathrm{mol}^{-1}[15]$. Below $120 \mathrm{~K}$, magnetization increases rapidly for both samples, which is compatible with Curie-Weiss behavior. This is confirmed by the straight lines in plots of the inverse magnetic susceptibility below $120 \mathrm{~K}$ (Fig. 3)

$$
\left[\chi(T)-\chi_{0}\right]^{-1}=(T-\theta) / C_{m}
$$

from which the molar Curie constant $C_{m}$ and the CurieWeiss temperature $\theta$ are determined. It is evident that the susceptibility is significantly enhanced after hydrogen desorption. From the low temperature range $\theta$ adopts values $<10 \mathrm{~K}$, nearly compatible with zero, which means that there is no magnetic interaction between the Pt clusters. This shows that the clusters are isolated, which is consis-
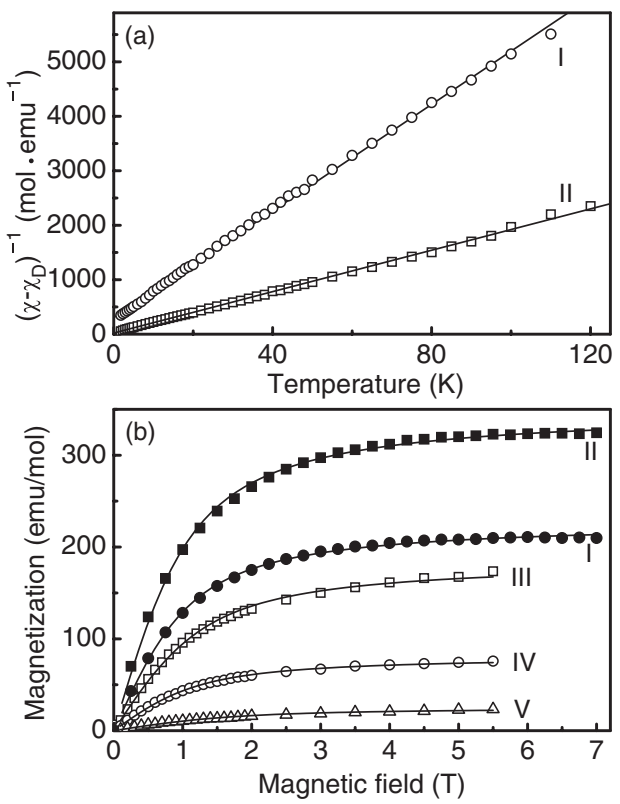

FIG. 3. (a) Inverse magnetic susceptibility (magnetization divided by field) per mol of of $\mathrm{Pt}$ atoms on $\mathrm{NaY}$ in the range of $1.8-120 \mathrm{~K}$ in an external field of $0.2 \mathrm{~T}$ before (I) and after hydrogen desorption (II); (b) Molar magnetization at $1.8 \mathrm{~K}$ of $\mathrm{Pt} / \mathrm{NaY}$ before and after hydrogen desorption, compared with literature data for analogous measurements of Pt nanoparticles embedded in a poly- $N$-vinyl-2-pyrrolidone (PVP) polymer [5]. The particles have diameters of $2.3 \mathrm{~nm}$ (ca. 420 atoms, III), $3.0 \mathrm{~nm}$ (940 atoms, IV) and $3.8 \mathrm{~nm}$ (V, 1900 atoms). tent with the fact that on the basis of a cluster size of 13 atoms only one out of 25 supercages of $\mathrm{NaY}$ is occupied.

At low fields, the effective magnetic moment $\mu_{\text {eff }}$ per Pt atom is related to $C_{m}$, the inverse slope of the lines in Fig. 3(a),

$$
C_{m}=N \mu_{\mathrm{eff}}^{2} / 3 k_{B}=N g^{2} J(J+1) \mu_{B}^{2} / 3 k_{B}
$$

This yields $\mu_{\text {eff }}$ per Pt atom as $0.45(5) \mu_{B}$ [corresponding to a $J \approx 2.9(3)$ state for a 13 atom cluster] and $0.65(5) \mu_{B}$ $[J \approx 4.2(3)]$ for the hydrogen-covered and the hydrogendesorbed samples, respectively. These values are far larger than the ones reported for the embedded Pt nanoparticles in a polymer, which amount to $0.012 \mu_{B} /$ atom $(2.3 \mathrm{~nm}$ diam $)$, $0.006 \mu_{B} /$ atom $(3.0 \mathrm{~nm})$, and $0.002 \mu_{B} /$ atom $(3.8 \mathrm{~nm})$ [5]. The values are in qualitative agreement with the theoretically predicted $S=4$ ground state that corresponds to $0.62 \mu_{B} /$ atom for the icosahedral, and $S=3$ $\left(0.46 \mu_{B} /\right.$ atom $)$ for the cuboctahedral bare neutral 13 atom Pt clusters [3]. For neutral clusters with adsorbed hydrogen, a ground state of $1 \leq S \leq 4$, depending on the degree of coverage, was predicted [4]. Although within error the observed effective moments translate into integer values of $S$, the samples may contain mixtures of clusters with different spin states. Furthermore, magnetization due to spin moments may be significantly enhanced by contributions due to atomic or overall cluster orbital moments which were not taken into account in the calculations. We therefore use the more general symbol $J$ for the experimental states.

The quantitative values derived above for $J$ depend on the value taken for the temperature-independent susceptibility $\chi_{0}$, which is subject to some uncertainty. For the present evaluation $\chi_{0}$ was chosen to yield an optimum fit to Eq. (1) for $T<120 \mathrm{~K}$. These values $\left(+3 \times 10^{-4} \mathrm{emu} \cdot\right.$ $\mathrm{mol}^{-1}$ for the hydrogen-reduced and $+6 \times 10^{-4} \mathrm{emu}$. $\mathrm{mol}^{-1}$ for the hydrogen-desorbed sample), are somewhat below the residual values of $\chi$ at $300 \mathrm{~K}$. Using the $300 \mathrm{~K}$ $\chi_{0}$ values would result in an even larger magnetic moment per Pt atom, but a fit of Eq. (1) using these $\chi_{0}$ values is not satisfactory in the temperature range up to $120 \mathrm{~K}$. This means that the present model assuming simple CurieWeiss behavior and involving a temperature-independent value of $\chi_{0}$ is somewhat too simple. Nevertheless, independent of the choice of $\chi_{0} \mu_{\text {eff }}$ is an order of magnitude higher than for the larger nanoparticles.

The field dependence of magnetization in Fig. 3(b) fits to the classical Langevin function,

$$
M(H)=N \mu\left[\operatorname{coth}\left(\frac{\mu H}{k_{B} T}\right)-\frac{k_{B} T}{\mu H}\right]+\chi_{0} H,
$$

where $N$ is the number of Pt clusters per unit volume, $T$ the temperature, $H$ the magnetic field, and $\mu$ the magnetic moment per cluster. No hysteresis is observed even at $1.8 \mathrm{~K}$. It is again obvious that the saturation magnetization is considerably higher after hydrogen desorption. The decrease in saturation magnetization with increasing particle 
size is qualitatively in agreement with expectation based on the increasing average Pt coordination number as conditions approach those of the bulk. However, it is interesting to note that on the basis of the results obtained from the susceptibility measurements at $0.2 \mathrm{~T}$ [Fig. 3(a)] the absolute saturation values are too low by as much as an order of magnitude, far beyond any error, corresponding to values of $0.0059 \mu_{B} /$ atom and $0.0038 \mu_{B} /$ atom for the hydrogendesorbed and hydrogen-reduced samples, respectively. This point is under further investigation, but it is already clear that the magnetization is quenched in high fields, perhaps through further lifting of the near-degenerate HOMO states with field. It could point to a highly interesting pseudoatom character of the $\mathrm{Pt}_{13}$ cluster.

The samples also give rise to an EPR spectrum that shows a multiplet due to a cluster of 12 equivalent $\mathrm{Pt}$ atoms with perhaps an invisible 13th atom [4]. The cluster size is compatible with the present EXAFS measurements, but this signal accounts for $<1 \%$ of the total Pt in the sample. From certain shifts of equilibria it was concluded that there must be a large reservoir of EPR-silent clusters of the same size [4]. The present work identifies this reservoir to consist of high-spin Pt clusters which are not observable via $X$-band EPR.

A few more comments about the observed novel magnetic behavior are in order. (i) The error in the EXAFS characterization of size dispersion is \pm 2 atoms. However, due to the extra stability of the geometric closed shell structure it is not unlikely that the clusters consist nearly quantitatively of 13 atoms, a magic number. The magnetic properties represent in any case an average over the given size distribution. (ii) The presence of high spin states with up to eight unpaired electrons per cluster $(S \leq J \approx 5)$ demonstrates that the clusters are of high symmetry with many molecular orbitals differing in energy by less than the spin pairing energy. Icosahedral symmetry permits degeneracies $S \leq 5$ which may be lifted by small structural distortions. Density functional calculations predict excited states within a few $\mathrm{kJ} \cdot \mathrm{mol}^{-1}$ of the ground state for some of the species [2-4]. Such low values are beyond the accuracy of these calculations and should not be overinterpreted. However, they show that the presence of low-lying excited states is highly probable also from the viewpoint of theory. (iii) A theoretical study of $\mathrm{H}$ adsorption at the surface of icosahedral $\mathrm{Pd}_{13}$ clusters showed that $1 \mathrm{H}$ per threefold face reduces the magnetic moment by $1 \mu_{B}$ resulting in a complete quenching of the magnetic moment [16]. The predicted decrease with $\mathrm{H}$ adsorption is in line with the present observations. $\mathrm{H}$ adsorption induces new states at around $6 \mathrm{eV}$ below the HOMO [16], and the formation of a chemical bond to each additional $\mathrm{H}$ depletes the HOMO by one electron. It is plausible that this happens also with $\mathrm{Pt}_{13}$, and it is conceivable that the cluster magnetic moment oscillates as a function of hydrogen coverage when successive energy levels are depleted, which should permit a "titration" of energy levels. The extent of coverage of the clusters is not known for the present experiments. (iv) A clarification of the orbital contribution to magnetization using $\mathrm{x}$-ray magnetic circular dichroism and the exact dependence of magnetization on hydrogen coverage and on magnetic field will be subject to further investigations.

In molecular beam experiments [1] the cluster mass is known with higher precision and the (unknown) structures are free of perturbation by a support, but the symmetry of these present open shell systems is expected to be broken also in the absence of a support. The advantages of the supported clusters are their accurate structural characterization and their potential application for advanced materials. It is anticipated that the unprecedented magnetic properties of these clusters will trigger theoretical as well as further experimental work.

$X$. Liu thanks the International Max Planck Research School for Advanced Materials (IMPRS-AM) for financial support.

*Corresponding author.

Email address: e.roduner@ipc.uni-stuttgart.de

[1] I. M. L. Billas, A. Chatelain, and W. de Heer, Science 265, 1682 (1994).

[2] M. Moseler, H. Häkkinen, R. N. Barnett, and U. Landman, Phys. Rev. Lett. 86, 2545 (2001).

[3] N. Watari and S. Ohnishi, Phys. Rev. B 58, 1665 (1998).

[4] X. Liu, H. Dilger, R. A. Eichel, J. Kunstman, and E. Roduner, J. Phys. Chem. B 110, 2013 (2006).

[5] Y. Yamamoto, T. Miura, Y. Nakae, T. Teranishi, M. Miyake, and H. Hori, Physica (Amsterdam) 329B333B, 1183 (2003).

[6] Y. Nakae, Y. Seino, T. Teranishi, M. Miyake, S. Yamada, and H. Hori, Physica (Amsterdam) 284B-288B, 1758 (2000).

[7] R. Kubo, J. Phys. Soc. Jpn. 17, 975 (1962).

[8] S. H. Yang, D. A. Drabold, J. B. Adams, P. Ordejón, and K. Glassford, J. Phys. Condens. Matter 9, L39 (1997).

[9] J. de Graaf, A. J. van Dillen, K.P. de Jong, and D.C. Koningsberger, J. Catal. 203, 307 (2001).

[10] M. Newville, P. Livins, Y. Yacoby, J. J. Rehr, and E. A. Stern, Phys. Rev. B 47, 14126 (1993).

[11] B. S. Clausen, L. Gråbæk, H. Topsø, L. B. Hansen, P. Stoltze, J. K. Nørskov, and O.H. Nielsen, J. Catal. 141, 368 (1993).

[12] A. Jentys, Phys. Chem. Chem. Phys. 1, 4059 (1999).

[13] A. I. Frenkel, C. W. Hills, and R. G. Nuzzo, J. Phys. Chem. B 105, 12689 (2001).

[14] F. W. Lytle, R. B. Greegor, E. C. Marques, D. R. Sandstrom, G. H. Via, and J.H. Sinfelt, J. Catal. 95, 546 (1985).

[15] H. Kojima, R. S. Tebble, and D. E. G. Williams, Proc. R. Soc. A 260, 237 (1961).

[16] V. Kumar and Y. Kawazoe, Phys. Rev. B 66, 144413 (2002). 